\title{
BLUE STRAGGLERS IN THE CORE OF THE GLOBULAR CLUS- TER 47 TUCANAE
}

\author{
G. Meylan, F. Paresce, and M. Shara \\ Space Telescope Science Institute \\ 3700 San Martin Drive \\ Baltimore MD 21218 U.S.A.
}

\begin{abstract}
High resolution imaging observations of the core of the globular cluster 47 Tucanae with the Faint Object Camera (FOC) on the Hubble Space Telescope (HST) reveal a very high surface density of blue stragglers. This discovery supports the hypothesis that they are among the most massive objects in globular clusters, concentrated in the core by mass segregation. Taken together with the presence in 47 Tuc of an X-ray source, eleven millisecond and binary pulsars, and two high-velocity stars ejected out of the core, these observations favour the scenario that blue stragglers are either mergers formed through coalescent collisions, or binaries formed through close encounters.
\end{abstract}

\section{INTRODUCTION}

Stellar densities in the cores of globular clusters are orders of magnitude higher than in the solar neighborhood. Close encounters with tidal interactions and physical collisions between stars should be commonplace in the densest parts of all but the sparsest globular clusters. The products of such events may include cataclysmic and low mass X-ray binaries, massive disk stars, and blue stragglers. The latter correspond to a sparse upward extension of the main sequence (MS) above the cluster turn-off and are situated on a locus of the color-magnitude diagram (CMD) previously occupied by stars which have now evolved off the MS. The three most probable scenarios for their formation are: coalescence after direct stellar collision, an extended MS lifetime due to mass transfer in binaries and an extended MS lifetime due to internal mixing in single stars.

\section{THE OBSERVATIONS}

The high spatial resolution and ultraviolet sensitivity of HST make it a potentially powerful tool for probing the dense centers of globular clusters. The data presented here were obtained on November 16,1990 with the FOC in its F/96 mode and on November 28,1990 in the F/48 mode. The telescope was pointed at the nominal optical center of 47 Tuc. The images taken around the internal best focus position of the F/96 camera yield a point spread function (PSF) of about $0.07^{\prime \prime}$ FWHM 
while the corresponding F/48 images show a $0.16^{\prime \prime}$ FWHM. The encircled energy within a $0.1^{\prime \prime}$ radius of the central peak corresponds to $15 \%$ of the total energy for the F/96 images and $9.5 \%$ of the total energy for the F/48 images at these wavelengths. The rest of the light is spread out over a circular halo of about $2^{\prime \prime}$ radius due to the spherical aberration of the primary HST mirror.

The raw images were flattened and geometrically corrected to remove the optical and detector distortions. In order to maximize the $\mathrm{S} / \mathrm{N}$ ratio of faint stars, several of the best frames were then registered and coadded. Thus, the final $\mathrm{F} / 48-$ F220W image used in this investigation corresponds to an effective exposure time of 27.8 minutes, and the final F/48-F140W image to 55.3 minutes.

Figure 1. F/48-F220W image of the core of 47 Tuc. The field is $22^{\prime \prime} \times 22^{\prime \prime}$ in size. The letters $\mathrm{A}, \mathrm{M}$, and $\mathrm{G}$ mark three positions of the cluster center found in the literature. $X$ marks the approximate center of the X-ray source error box. The numbered objects are the newly discovered blue stragglers.

\section{PHOTOMETRIC RESULTS}

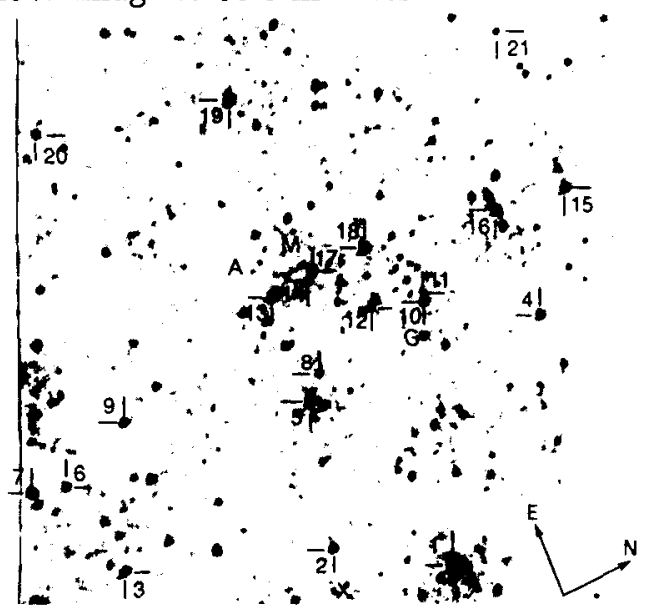

Standard photometry routines were used to compute the fluxes of about 125 stars in the F/48-F140W and F220W frames. The results are shown in Figure 2 in the form of a UV CMD in which $\mathrm{m} 220$ is plotted vs. $\mathrm{m} 140-\mathrm{m} 220$. With the quoted exposure times and efficiencies, the 5 sigma threshold applied to the data corresponds to a limiting m220 magnitude of about 20 . Factoring in all the uncertainties involved, we estimate a 1 sigma error of \pm 0.1 magnitude in $\mathrm{m} 220$ and $\mathrm{m} 140$ for stars brighter than 19 growing to a few tenths of a magnitude for fainter stars.

The content of our unusual UV CMD can be understood by mapping the visual CMD into the UV CMD with the help of its corresponding visual CMD determined from ground-based observations. This is done by running the known spectra of stars of the appropriate luminosity class and spectral type through the FOC instrument simulation software FOCSIM. In general, the redder the star the more negative the corresponding m140-m220 color owing to the slightly higher transmissivity of the F140W filter beyond about $5000 \AA$.

Most of the stars of the sample shown in Figure 2 lie to the right of the origin and cluster around the mean positions of the known structures of 47 Tuc's CMD. The bright stars to the left of the origin, on the other hand, constitute a well defined population of about 21 stars brighter than $\mathrm{m} 220=19$ with a definite positive $\mathrm{m} 140$ $\mathrm{m} 220$ color. Their red color here simply reflects the fact that they are significantly fainter below $2000 \AA$ than above. They are identified by their numbers in the CMD of Figure 2 and in the image shown in Figure 1. They cannot be explained as a result of any known stellar population in the Hesser et al. (1987) visual CMD. 
Figure 2 The UV color magnitude diagram of the stars detected in both the $\mathrm{F} / 48-\mathrm{F} 220 \mathrm{~W}$ and -F140W fields. The solid lines represent the expected locations of the red giant (RGB), asymptotic giant (AGB), red horizontal (RHB) and main sequence (MS) branches in Hesser et al. (1987) mean composite visual color magnitude diagram. The blue stragglers are marked in accordance with Figure 1.

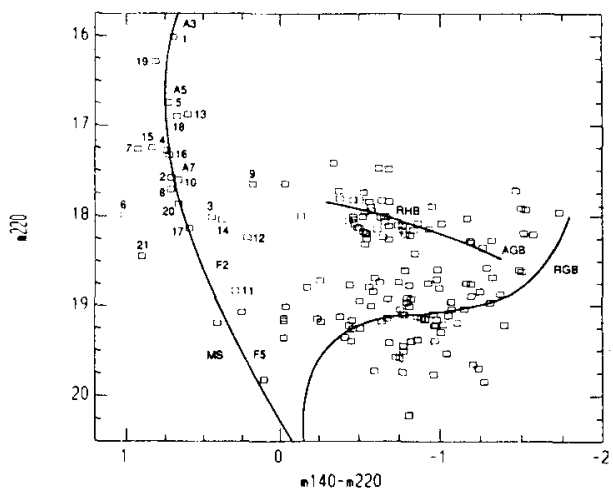

\section{BLUE STRAGGLERS IN THE CORE}

Hardly any of the 21 HST blue stars have appreciable flux in our ground based B,V frames. The excellent match in Figure 2 between observed and simulated sequences allows us to conclude that most of these $21 \mathrm{UV}$ bright stars are blue stragglers. The visible flux from one source (HST-2) was measured by Aurière et al. (1989) who find $\mathrm{U}=16.73, \mathrm{~B}=16.83$, and $\mathrm{V}=16.01$ (\# 6 in their Table 6 ). This star is located inside the error circle of the position of the $\mathrm{X}$-ray source and is the object in the error box with by far the bluest $\mathrm{U}-\mathrm{B}$ index.

The high surface density of the blue stragglers we have discovered in the core is not maintained out to the periphery (Hesser et al. 1987). This gradient in the density of blue stragglers towards the core of 47 Tuc lends further weight to the hypothesis that blue stragglers are significantly more massive than cluster main sequence and subgiant stars. Because of the short relaxation time in the core of $47_{\mathrm{Tuc}} \sim 10^{7} \mathrm{yr}$, i.e., much less than the cluster age, $\sim 15 \mathrm{Gyr}$, mass segregation should have taken place through equipartition of energy, the most massive stars sinking towards the inner parts.

This population of possible binaries is interesting from the point of view some recent observations: apart from the $\mathrm{X}$-ray source, two high-velocity stars have been found in the core of 47 Tuc by Meylan et al. (1991), which could only be the result of gravitational interactions between single stars and binaries or between two binaries. There is also the recent discovery of eleven millisecond and binary pulsars which adds weight to the conjecture that binaries are present in this cluster, with their possible crucial role in the late phases of the cluster's dynamical evolution.

\section{REFERENCES}

Aurière, M., and Ortolani, S. 1988, AA, 204, 106.

Aurière, M., Koch-Miramond, L., and Ortolani, S. 1989, AA, 214, 113.

Bailyn, C.D., Grindlay, J.E., Cohn, H., and Lugger, P.M. 1988, ApJ, 331, 303.

Hesser, J.E., Harris, W.E., and Vandenberg, D.A. 1987, PASP, 99, 739.

Meylan, G., Dubath, P., and Mayor, M. 1991, ApJ, in press.

Paresce, F., Shara, M., Meylan, G., and the FOC IDT 1991, Nat, 84, 752. 